\title{
Another type of log-periodic oscillations on Polish stock market?
}

\author{
Piotr Gnaciński*and Danuta Makowiec $^{\dagger}$ \\ Institute of Theoretical Physics and Astrophysics, Gdańsk University \\ ul.Wita Stwosza 57, 80-952 Gdańsk, Poland
}

November 10, 2018

\begin{abstract}
Log-periodic oscillations have been used to predict price trends and crashes on financial markets. So far two types of log-periodic oscillations have been associated with the real markets. The first type are oscillations which accompany a rising market and which ends in a crash. The second type oscillations, called "anti-bubbles" appear after a crash, when the prices decreases. Here, we propose the third type of log-periodic oscillations, where a exogenous crash initializes a log-periodic behavior of market, and the market is growing up. Such behavior has been identified on Polish stock market index between the "Russian crisis" (August 1998) and the "New Economy crash" in April 2000.
\end{abstract}

Key words: Econophysics; Stock market; Log-periodic oscillations; Power laws; Crashes

\section{Introduction}

Starting from 1996, Sornette et al. [1] in the series of papers [2, 3, 4, 5, 6, 7, 8, 9 10, give arguments that crashes are analogous to critical points, which are preceded by log-periodic oscillations. Such oscillations were studied in statistical physics. The interactions between investors lead to a speculative "bubble" which ends in a crash. Over 50 such crashes have been described on stock, FX and Gold market. Moreover, using the symmetric in time bubble formula they have found so called "anti-bubble" price development which describes how a market behaves after a crash. Starting at rapidly oscillating state after the crash a market goes down with its price decorated by log-periodic oscillations. Both types of oscillations: bubble and anti-bubble are assigned to herding behavior of investors. However difficulties have been encountered when crashes on Eastern

*pg@iftia.univ.gda.pl

†fizdm@univ.gda.pl 
Europe stock markets were studied [5]. We propose another type of oscillations, where an exogenous crash initializes a log-periodic price development, but the prices are rising. We will call it "inverted bubble".

In order to accurately define a crash we use so called "drawdowns". A drawdown is a persistent decrease in the price over consecutive days [8]. We ignore corrections between local maximum and local minimum less than $\epsilon=30 \%$ of the preceding price fall.

Fig.1 presents a scheme of a $\epsilon$-drawdown. In the first step we construct drawdowns (drawups) by merging together quotations with price changes in the same direction. For example, the first three prices of Fig.1. are merged into a drawdown $\mathrm{AB}$, the next two create a drawup $\mathrm{BC}$, followed by a drawdown $\mathrm{CD}$. In the second step $\epsilon$-drawdowns are constructed as follows.If the drawup BC: $P_{C}-P_{B}$, is less than $\epsilon$ (here we assume that $\epsilon=30 \%$ ) of the absolute value of the drawdown $\mathrm{AB}:\left|P_{B}-P_{A}\right|$, and price in the point $\mathrm{D}$ is less than in the price in the point $\mathrm{B}$, than the drawup $\mathrm{BC}$ is treated as the correction to the trend. So that the $\epsilon$-drawdown $\mathrm{AD}$ is made in place of the two drawdowns $\mathrm{AB}$, and $\mathrm{CD}$ and the drawup BC. Finally, the price change is transferred into logarithms:

$$
\text { drawdown }=\ln \left(P_{\max } / P_{\min }\right)
$$

where $P_{\max }$ and $P_{\min }$ are prices at beginning and at the end of a drawdown (here points A and D). According to [8] the distribution of drawdowns is a stretched exponential

$$
f(x)=a \cdot \exp \left(-b|x|^{z}\right)
$$

Fig.2 shows the distribution of drawdowns of WIG (Warsaw Stock Index) from 1991 to August 2002. The fit of stretched exponential to this distribution gives us: $a=63.6, \quad b=0.019, \quad z=1.08$. Hence, events with drawdowns less than $-14.5 \%$ are outliers. We will adopt a definition of a crash as a drawdown with price loss over $14.5 \%$. An extreme rise will be a drawup of more than $17.5 \%$.

\section{Log-periodic oscillations}

The prices or index values prior to crash are described by the first term "Landau" expansion. The expansion describes a power law behavior of price $p(\tau)$ :

$$
\frac{d p(\tau)}{d \ln \tau}=(\beta+i \omega) p(\tau)
$$

This equation is sometimes written in the form:

$$
\frac{d \ln p(\tau)}{d \ln \tau}=(\beta+i \omega)
$$

which illustrates that the price $p(\tau)$ becomes self-similar with respect to the dilation of the distance $\tau$. The relative variations $d \ln p=d p / p$ of price with 
respect to the relative variations of the time to crash $d \ln \tau=d \tau / \tau$ are independent of time. From equation 3 one can obtain a log-periodic development of price:

$$
p(t)=A+\tau^{\beta} \cdot[B+C \cos (\omega \ln \tau+\phi)]
$$

where $\tau=\left|t_{c}-t\right|$, and $t_{c}$ is critical time (the time of crash).

The numerical way to identify log-periodicity in a time series is to fit the 7 parameters-function from eq. 5 to the data. The amoeba fitting procedure [1] was used to minimize the variance:

$$
V a r=\frac{1}{N} \sum_{k=1}^{N}\left(p_{k}-p\left(t_{k} ; A, B, C, \beta, \omega, t_{c}, \phi\right)\right)^{2}
$$

Where $\mathrm{N}$ is the number of points in the data set, $t_{k}$ and $p_{k}$ are the time and price of the $\mathrm{k}$-th point.

To convince that the oscillations are really present in the data we transform the analyzed data to a pure cosine function [7:

$$
\begin{array}{r}
t_{k} \longrightarrow \ln \left|t_{k}-t_{c}\right| \\
p_{k} \longrightarrow \frac{p_{k}-\left(A+B \tau^{\beta}\right)}{C \tau^{\beta}}
\end{array}
$$

From the transformed data we made a Lomb periodogram [1] The Lomb periodogram is used to find periodicity in unevenly sampled data. The Lomb periodogram should give us the same frequency as the fitting procedure.

At least four distinct log-oscillation events can be identified on the Polish stock market index WIG in the years 1991 to 2002 (Fig. 3): the speculative bubble which ended with the crash in March 1994, then an anti-bubble recovery from that crash and the inverted bubble crash of April 2000 followed by the anti-bubble price development.

\section{Why inverted log-periodic oscillation?}

The Nasdaq "New Economy" fall in April 2000 was a bubble ending in a crash 3. This event is an instance of log-periodic price development on rising market ending with a crash. A fit with eq. (5) describes the behavior of Nasdaq from Spring 1997 to April 2000. The examined period consists of four evident oscillations.

The "New Economy" crash was also observed on the Polish stock market. However no satisfying long-term fit with the eq. (5) could be made. Here are our attempts. On Fig.4 the bubble fit to Warsaw Stock Index WIG is shown. The best fit extends over one year, with one oscillation period only, and gives the critical time 4 months after the "New Economy" crash happened. On Fig. 5 the fit to the period August 1998 (so called "Russian crisis", which caused WIG drawdown $=-31.5 \%$ ) to August 2000 is shown. The critical time is April 2000, but the fit is based on a small correction in January 2000, which itself could not 
be well approximated. It is impossible to predict this crash with log-periodic function. It was possible to made the presented fit only because we know when the crash occurred. Summing up, we claim that both fits are doubtful, and are extended only about 1.5 periods of the cosine function.

Therefore we propose another possibility of describing the development of WIG index in the time period from August 1998 to April 2000. On August 1998 the "Russian crisis" occurred. The "Russian crisis" was a exogenous event for the Polish stock market. The critical time $t_{c}$ (August 1998) means the starting point of inverted bubble: a log-periodicity price behavior with a rising market. Now, the fit extends over 4 periods of the cosine function (Fig. 6). We have found two satisfactory fits to the WIG index: the first fit is with $\omega=9.8$ and second one is with $\omega=11.3$.

Unfortunately, the inverted bubble was not found in Nasdaq data. From an inverted bubble one can not predict the time of the next crash (April 2000 in this case). However it can be expected, that crash should coincide with the maximum of the cosine function.

\section{Note}

After finishing this paper we have recognized that inverted bubbles were independently described by Zhou and Sornette 12. They have found six market indices with such behavior (they call them "bullish anti-bubbles"). All critical times were set between August and November 2000. However, these log-periodic oscillations have not ended with a crash yet.

\section{Acknowledgments}

This work was supported by Gdańsk University grant BW 5400-5-0014-3.

\section{References}

[1] Sornette, D., Johansen, A., Bouchaud J.P., J.Phys.I France 6, 167-175 (1996)"' sStock market crashes, Precursors and Replicas"'

[2] Sornette, D., Johansen, A., Physica A 245, 411, (1997), "Large financial crashes"

[3] Johansen, A., Sornette, D., EPJ B 17, 319-328, (2000), "The Nasdaq crash of April 2000: Yet another example of log-periodicity in a speculative bubble ending in a crash"

[4] Johansen, A., Sornette, D., Int.J.Mod.Phys.C 10(4), 563-575, (1999), "Financial 'Anti-Bubbles': Log-Periodicity in Gold and Nikkei collapses"'

[5] Johansen, A., Sornette, D., Int.J.Theor.and App. Finance 4 (6), 853-920 (2001), "Bubbles and anti-bubbles in Latin-American, Asian and Western stock markets: An empirical study" 


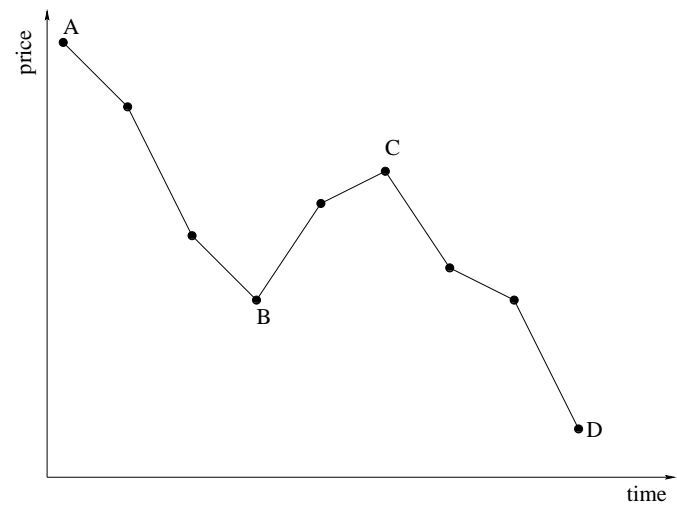

Figure 1: Construction of $\epsilon$-drawdowns.

[6] Johansen, A., Sornette, D., arXiv:cond-mat/0210509 "Endogenous versus Exogenous Crashes in Financial Markets"

[7] Johansen, A., Sornette, D., O. Ledoit, Journal of Risk, vol. 1, number 4, 5-32 (1999), "Predicting Financial Crashes Using Discrete Scale Invariance"

[8] Johansen, A., arXiv:cond-mat/0205249, (2002),"Comment on: Are financial crashes predictable?"

[9] Johansen, A., Sornette, D., EPJ B 1, 141, (1998), "Stock market crashes are outliers"

[10] Johansen, A., Sornette, D., arXiv:cond-mat/9901035, "Critical Crashes"

[11] Press, W., Flannery, B., Teukolsky, S., Vatterling, W., "Numerical Recipes in C: the art of scientific computing", 2nd ed., Cambridge, Cambridge Univ. Press, (1992)

[12] Zhou W.-X., Sornette, D., arXiv:cond-mat/0212010, "Evidence of a Worlwide Stock Market Log-Periodic Anti-Bubble Since Mid-2000" 


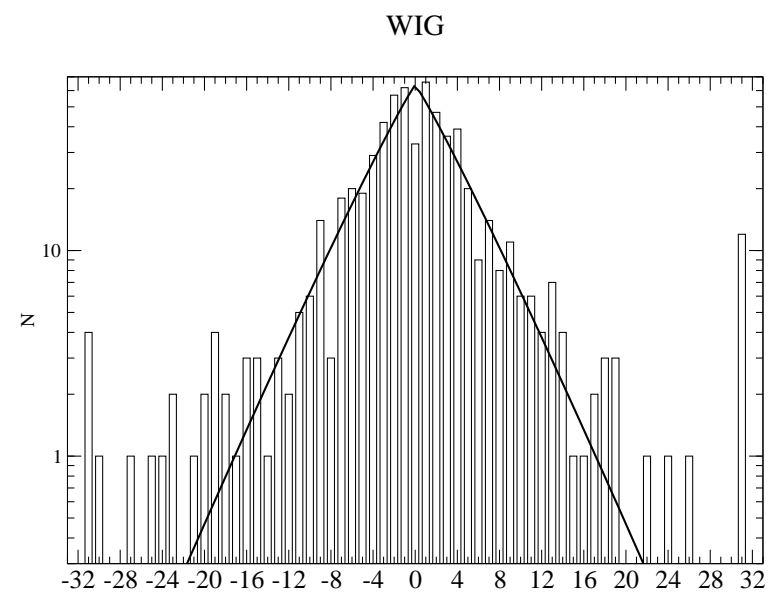

Figure 2: The histogram of drawdowns and drawups (in \%) on on Polish stock market index WIG from beginning (1991) to August 2002. The solid line is a best fit to the distribution with equation $2: a=63.6, b=0.019, z=1.08$. 


\section{WIG}
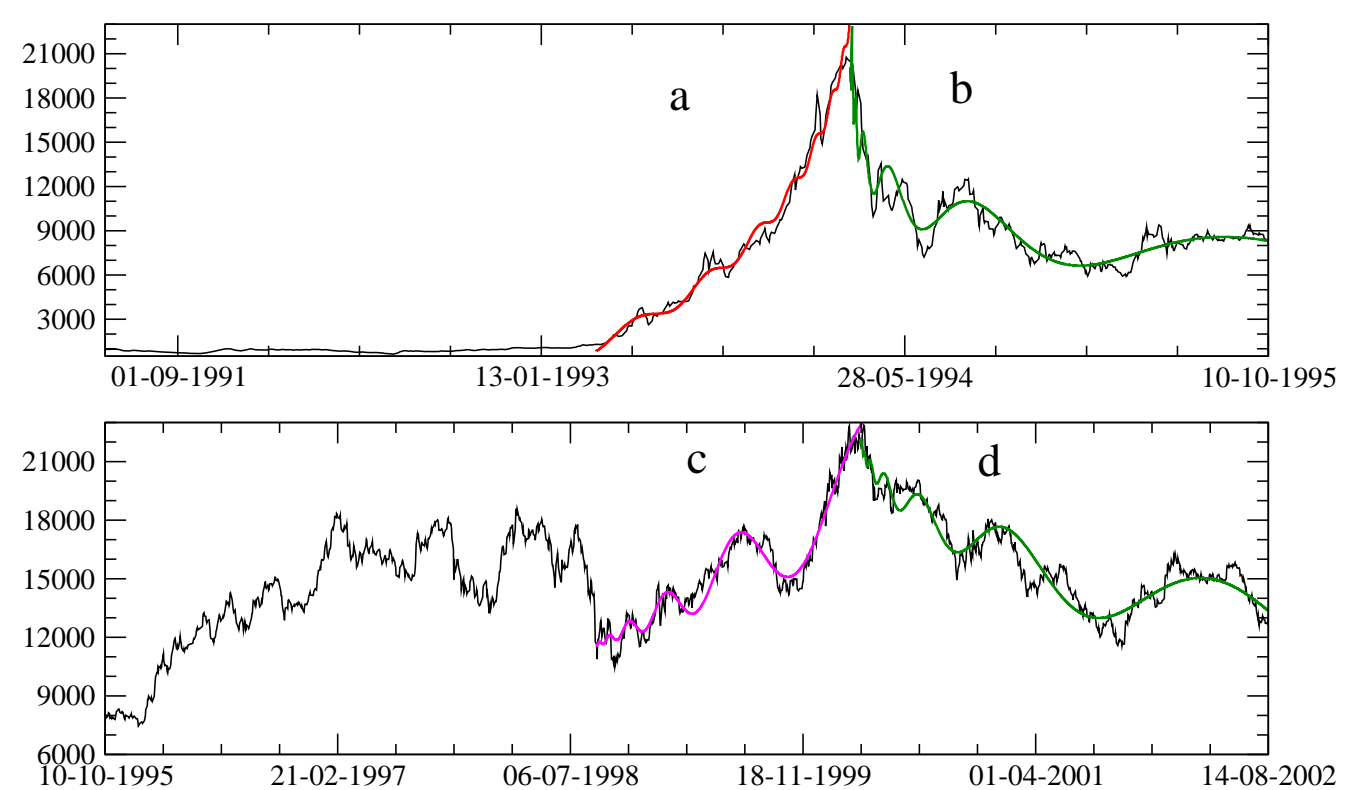

Figure 3: Two endogenous crashes observed on Polish stock market index WIG. The symbol a marks a bubble, the symbol $\mathbf{c}$ marks an inverted bubble, and $\mathbf{b}$ and $\mathbf{d}$ are for anti-bubbles. 


\section{WIG}
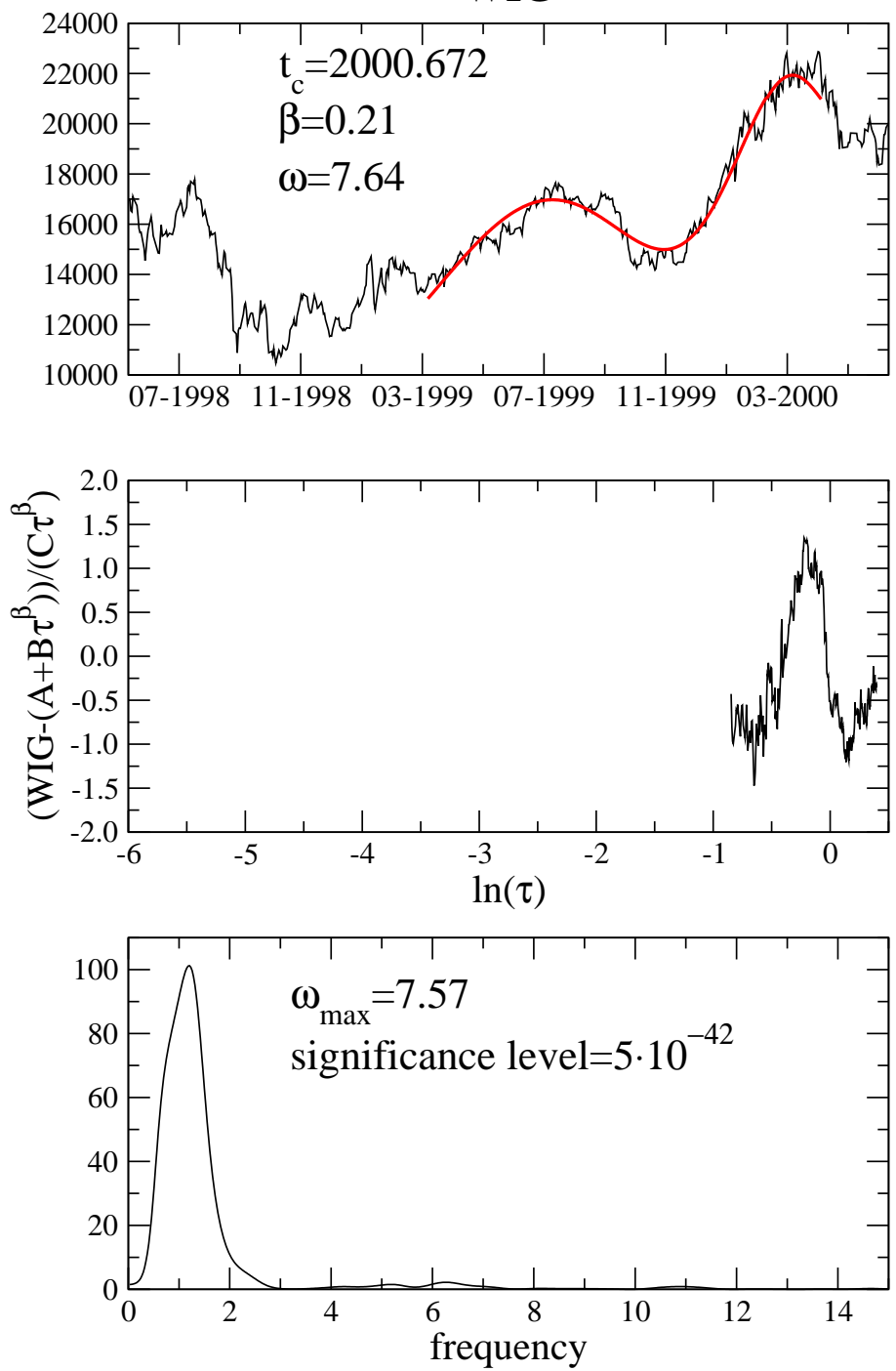

Figure 4: Log-periodicity fit to WIG data with crash 4 months after April 2000: $\beta=0.21, \omega=7.64, t_{c}=2000.672$. The middle panel shows the data transformed to the cosine function with aid of equations (7) and (8). The bottom panel is the Lomb periodogram. 


\section{WIG}
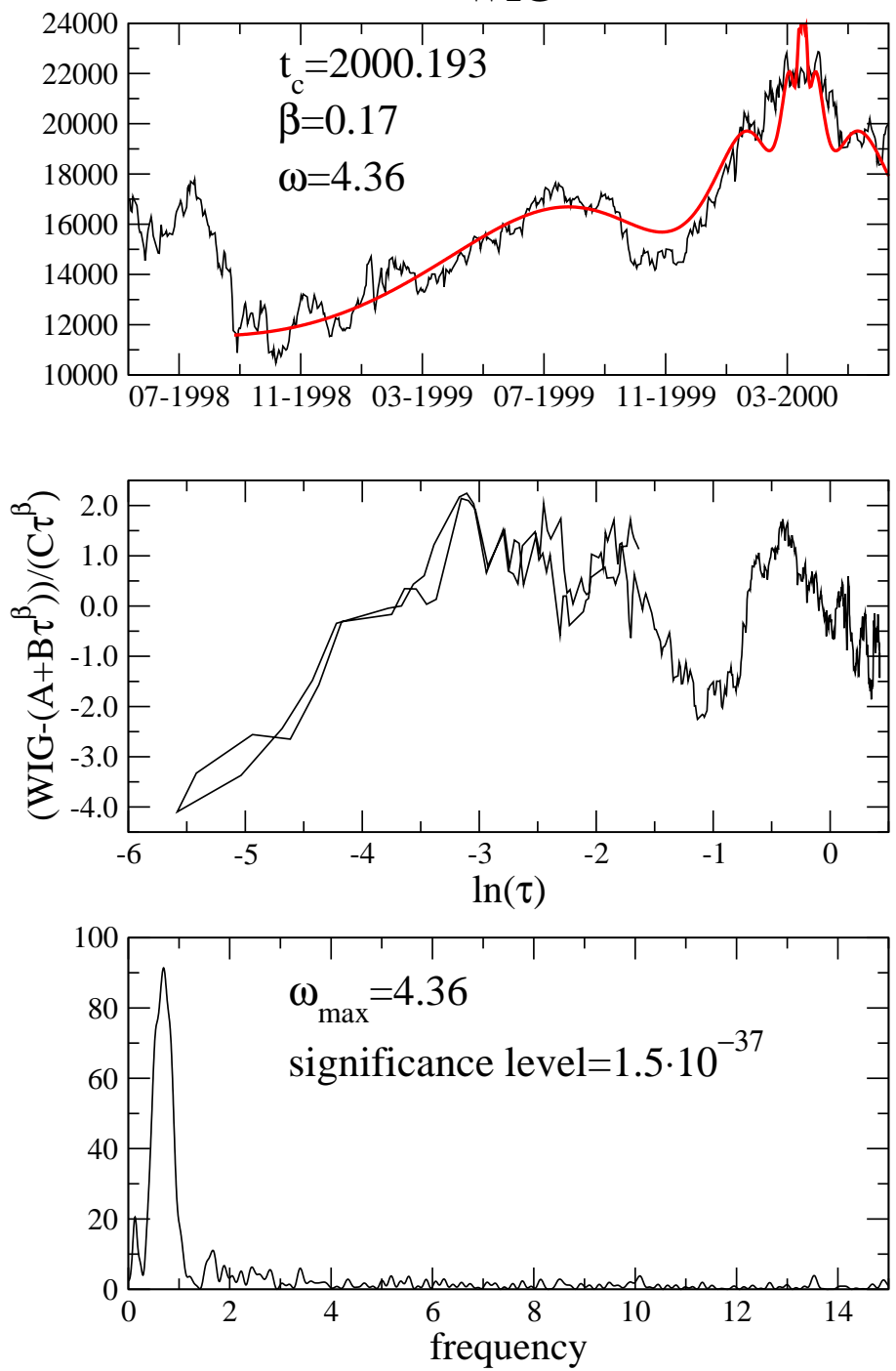

Figure 5: A log-periodicity fit to WIG with critical time in April 2000: $\beta=$ $0.17, \omega=4.36, t_{c}=2000.193$. The middle panel shows the data transformed to the cosine function with aid of equations (7) and (8). The bottom panel is the Lomb periodogram. 


\section{WIG}
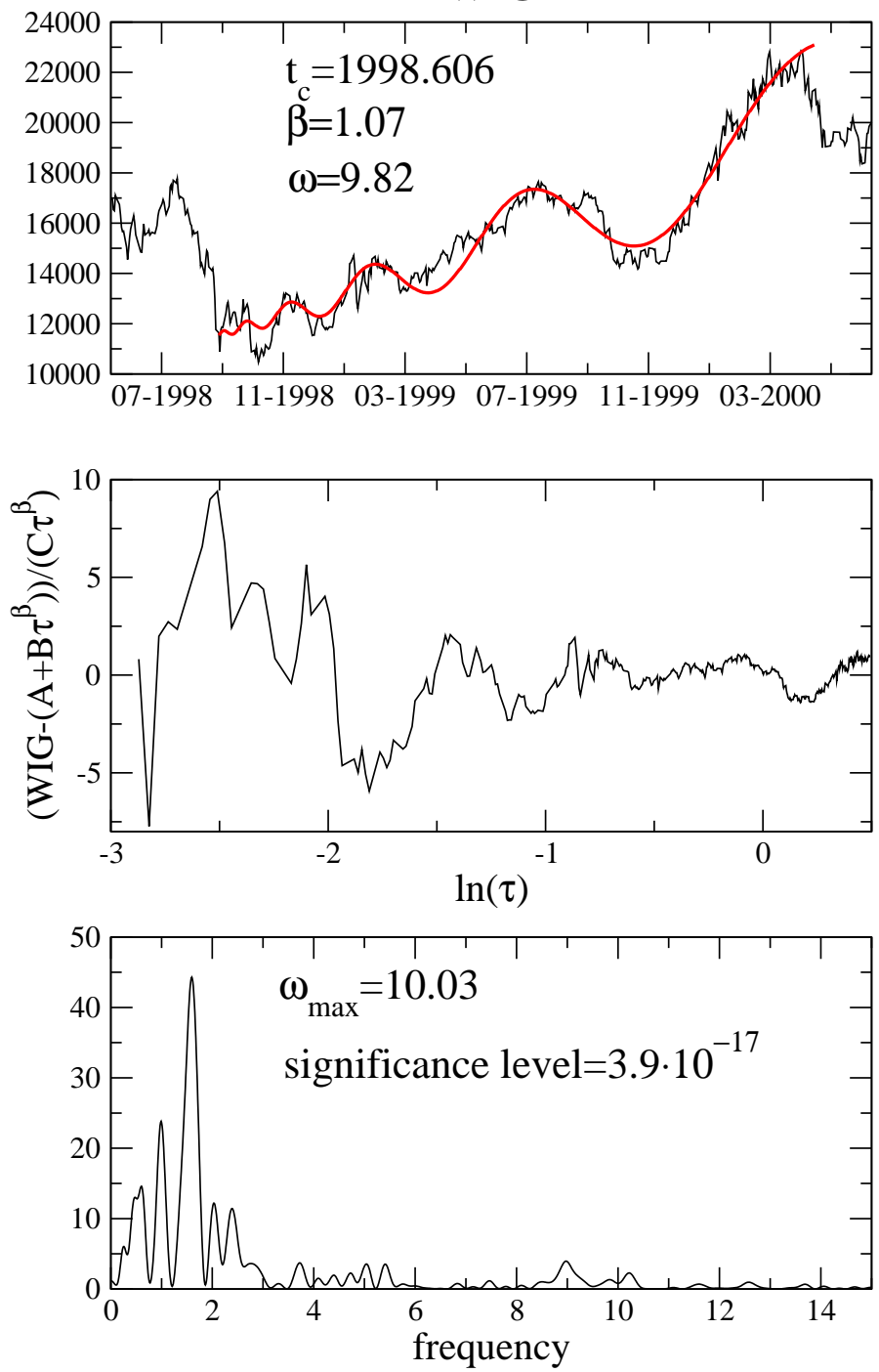

Figure 6: Inverted bubble for the Polish stock index WIG: $\beta=1.07, \omega=$ $9.82, t_{c}=1998.606$. Notice, that the fit expands over 4 periods of the cosine function. The middle panel shows the data transformed to the cosine function with aid of equations (7) and (8). The bottom panel is the Lomb periodogram. 